\title{
The Development and Future of Digestive Tract Reconstruction after Distal Gastrectomy: A Systemic Review and Meta-Analysis
}

\author{
Xi-Jie Chen ${ }^{1,2^{*}}$, Yun-Zhi Chen ${ }^{3^{*}}$, Dong-Wen Chen ${ }^{1,2^{*}}$, Ying-Liang Chen ${ }^{1,2^{*}}$, Jun Xiang ${ }^{1,2}$, Yi-jia Lin ${ }^{1,2}$, Shi \\ Chen $^{1,2}$, Jun-Sheng Peng $1,2 \bowtie$ \\ 1. Guangdong Provincial Key Laboratory of Colorectal and Pelvic Floor Diseases, The Sixth Affiliated Hospital, Sun Yat-Sen University, Guangzhou, \\ Guangdong 510655, P. R. China. \\ 2. Department of Gastrointestinal Surgery, The Sixth Affiliated Hospital, Sun Yat-Sen University, Guangzhou, 510655, P. R. China. \\ 3. Department of Endocrinology and Metabolism, Third Affiliated Hospital of Sun Yat-Sen University, and Guangdong Provincial Key Laboratory of \\ Diabetology, Guangzhou, 510630, P. R. China. \\ *These four authors contributed equally to this manuscript.
}

$\triangle$ Corresponding authors: Jun-Sheng Peng: The Sixth Affiliated Hospital, Sun Yat-Sen University, No 26, Yuancun Erheng Road, Tianhe District, 510655, Guangzhou, P. R. China. Phone:+86.20.3825.4092; Fax:+86.20.3825.4092; E-mail: pengjunsheng@tom.com and Shi Chen: The Sixth Affiliated Hospital, Sun Yat-Sen University, No 26, Yuancun Erheng Road, Tianhe District, 510655, Guangzhou, P. R. China. Phone: +86.20.3825.4092; Fax: +86.20. 3825.4092; E-mail: cscp@163.com

(c) Ivyspring International Publisher. This is an open access article distributed under the terms of the Creative Commons Attribution (CC BY-NC) license (https://creativecommons.org/licenses/by-nc/4.0/). See http://ivyspring.com/terms for full terms and conditions.

Received: 2018.07.29; Accepted: 2018.12.09; Published: 2019.01.24

\begin{abstract}
Background: Billroth I, Billroth II, Roux-en-Y, and Un-cut Roux-en-Y are common reconstruction techniques of distal gastrectomy. Which of these techniques is better has yet to be established. We performed an indirect comparison to evaluate which technique was optimal for preventing reflux symptoms.

Methods: The PubMed, Cochrane Collaboration, Embase, ClinicalTrials.gov and Web of Science databases were searched to identify clinical trials that compared at least two of the reconstruction skills among Billroth I, Billroth II, Roux-en-Y, and Un-cut Roux-en-Y. Data on reflux gastritis, intraoperative blood loss, bile reflux and postoperative hospital stays were extracted from the included clinical trials for meta-analysis using a random-effects model.

Results: Twenty-four articles that included 5419 individuals were assessed as eligible for meta-analysis. The indirect comparison suggested that Roux-en-Y reconstruction significantly reduces reflux gastritis, and it tended to rank first and had the highest probability of preventing bile reflux. No significant differences were found in intraoperative blood loss and postoperative hospital stays.

Conclusion: This indirect comparison suggested some superiority of Roux-en-Y reconstruction after distal gastrectomy. Further perspective clinical trials are required to provide evidence for the optimal reconstruction skill.
\end{abstract}

Key words: meta-analysis, distal gastrectomy, reconstruction, indirect comparison, net-work meta-analysis

\section{Introduction}

Radical distal gastrectomy is regarded as the standard surgery for the lower two thirds of gastric cancer patients. Previous studies have found that the extent of gastrectomy and systematic lymphadenectomy surgery greatly contribute to patient survival rates $(1,2)$. With the improvement of surgical skills and chemotherapy drugs, gastric cancer patients have obtained a more satisfying survival rate than ever before. As a result, postoperative quality of life (QOL) has been a concern for an increasing number of surgeons. However, the best reconstruction has remained controversial $(3,4)$.

Billroth I anastomosis (BI) has been accepted for years after it was first proposed by Theodor for gastric 
cancer patients. In the late 1990s, the highlights of BI were its technical simplicity, safety and physiological rationality of allowing chyme to directly pass the duodenum (5). However, for patients undergoing BI, reflux symptoms, such as heart burn, dyspnea, reflux gastritis, reflux esophagitis, bile reflux and so on, frequently occurred. Although Billroth II (BII) anastomosis can reduce anastomotic distention by BI, it does not change postoperative complications and leads to another challenge, dumping syndrome (6). To address this complication, Roux-en-Y (RY) reconstruction was explored because, in theory, it can relieve the bile reflux syndrome. However, RY also has complications, such as Roux limb stasis, internal hernia, and intestinal obstruction (7). Recently, some studies have suggested that BII with Braun anastomosis may effectively work as an anti-reflux technique, but prospective randomized studies have failed to prove this (8).

Uncut Roux-en-Y (URY) is a modified reconstruction technique based on BII and RY anastomosis. Some studies have confirmed that this type of reconstruction can preserve myoneural continuity to eliminate Roux stasis syndrome.(9-11) In previous studies, URY has been reported to reduce bile reflux disease and residual gastritis compared with BII $(12,13)$. Surgeons were delighted when they found that short-term outcomes indicated that URY reconstruction relieved anti-reflux symptoms and Roux stasis (14). However, many of the studies were retrospective studies. There is still a long way to go to prove that URY is a better choice for distal gastric cancer patients after gastrectomy compared with BII and RY anastomosis.

Hence, this indirect comparison among clinical trials was performed to assist clinical surgeons in making better decisions regarding the use of BI, BII, RY, or Uncut RY after performing radical distal gastrectomy.

\section{Materials and Methods}

The indirect comparison we performed strictly adhered to the preferred reporting for systematic reviews and meta-analyses (PRISMA) statement.

\section{Data retrieved and search strategy}

Published or unpublished comparisons of at least two digestive tract reconstruction skills among BI, BII, RY and URY after a distal radical gastrectomy were searched from the PubMed, Cochrane Collaboration, Embase, ClinicalTrials.gov and Web of Science databases. The search strategy of this indirect comparison was established for each database by using a combination of medical subject headings (MeSH) and free-text terms, as follows for
PubMed: ("Gastroenterostomy"[Mesh] OR "gastroenterostomies" [Title/Abstract] OR "BI procedure" [Title/Abstract] OR "procedure, Billroth" [Title/ Abstract] OR "BI operation" [Title/Abstract] OR "operation, BI" [Title/Abstract] OR "BI" [Title/ Abstract] OR "BII procedure" [Title/Abstract] OR "procedure, BII" [Title/Abstract] OR "BII operation" [Title/Abstract] OR "operation, BII" [Title/Abstract] OR "BII" [Title/Abstract]) OR ("Anastomosis, RY" [Mesh] OR "RY Loop" [Title/Abstract] OR “Loop, RY" [Title/Abstract] OR "Loops, RY" [Title/Abstract] OR "Roux en Y Loop" [Title/Abstract] OR "RY Loops" [Title/Abstract] OR "RY Anastomosis" [Title/ Abstract] OR "Anastomoses, RY" [Title/Abstract] OR "Roux en Y Anastomosis" [Title/Abstract] OR "RY Anastomoses" [Title/Abstract] OR "RY Diversion" [Title/Abstract] OR "Diversion, RY" [Title/Abstract] OR "Diversions, RY" [Title/Abstract] OR "Roux en $Y$ Diversion" [Title/Abstract] OR "RY Diversions" [Title/Abstract]). No language restriction was set in our research, and all related clinical trials, considering both RCTs or non-RCTs, were included. References were also searched for relative articles. Titles and abstracts were first used to select eligible articles, and then, full texts were used to make a final decision. Two researchers were responsible for this work, and they worked independently. A thorough discussion occurred or a senior researcher was recruited if an agreement was not reached. The last online search of our study was carried out on March 25, 2018.

\section{Inclusion criteria}

The inclusion criteria included: 1) patients who received radical distal gastrectomy; 2) a comparison of two or more reconstructions among BI, BII, RY and URY digestive tract reconstructions was mentioned in the study; and 3) at least one of the following outcomes was displayed: a postoperative hospital stay, intraoperative blood loss, reflux gastritis, and bile reflux. Relevant animal experiments and non-English publications were excluded.

\section{Data extraction}

Data from the included articles were extracted by two investigators separately according to our pre-designed data extraction forms. After a detailed discussion of the existing deviations with the senior investigator, we reached an agreement for each study. The information gathered from the articles were: authors, study design, duration time, sources of patients, reconstruction skills, numbers of patients, age, sex, length of hospital stay, blood loss, reflux gastritis, and bile reflux. For those studies that were repeatedly reported, we included the latest publication. Those studies whose research objects 
were not exactly the same were not excluded. For studies with incomplete data, we contacted the responding authors, and if the data were not available, the study was excluded.

\section{Outcome measures}

The primary outcome was reflux gastritis, and the secondary outcomes were intraoperative blood loss and bile reflux. In addition, the length of hospital stay after the operation was also used to evaluate the morbidity of perioperative events.

\section{Assessment of the methodological quality of the included studies}

The methodological quality of the included studies was assessed with two common quality evaluation tools. The Cochrane Collaboration's tool, which evaluates the risk of bias, was used to evaluate random sequence generation, allocation concealment, blinding of participants and personnel, blinding of outcome assessment, incomplete outcome data, selective reporting and other biases for RCTs. The risks were identified as low risk, unclear risk and high risk. The Newcastle-Ottawa Scale system (NOS) was used to evaluate the quality of non-RCTs according to their scores.

\section{Statistical analysis}

The data used in this meta-analysis were intended as treatments. Dichotomous data were analyzed to estimate the odds ratios (ORs) of each study for binary variables and the mean difference (MD) for continuous outcomes. Data were transformed into appropriate forms according to Stela Pudar Hozo et al. (15). Outcomes were presented with a 95\% confidence interval. Indirect comparisons were conducted using WinBUGS version 1.4.3 (MRC Biostatistics Unit, Cambridge, UK). We generated a random-effects model within a Bayesian framework, and to fit the model, we used three chains with over-dispersed initial values, with Gibbs sampling based on 20000 iterations after a burn-in phase of 50 000 iterations. ORs and MDs were compared to define the best probability related to each one being the best interventional factor when treatment effects were observed. The model we established enabled us to make direct and indirect comparisons of the studies, and when a direct comparison was not manageable, we still obtained an indirect comparison.

Review Manager 5.3 (Cochrane Collaboration, Oxford, UK) was used to present the results of the indirect comparisons and to conduct a traditional pairwise meta-analysis. $\mathrm{P}<0.05$ was considered statistically significant for all tests.

\section{Results}

\section{Study selection}

A total of 423 relevant articles were yielded by the research strategy in the initial search. Then, 35 potential articles were screened for full-text review, including 15 RCTs and 21 retrospective studies. One RCT using a BII reconstruction and Braun anastomosis was excluded. One RCT was excluded because of repeated publication. Another 2 RCTs were excluded because of no target endpoints, and 3 retrospective studies were also excluded. Moreover, 4 retrospective studies with three arms were excluded because multi-arm studies may artificially reduce the standard deviation. As a result, only pairwise studies of 10 RCTs $(7,16-24)$ and 14 retrospective studies (25-38) were eligible for final data extraction. The flow chart is shown in Figure 1. All studies examined at least one of the following outcomes: reflux gastritis, bile reflux, intraoperative blood loss and postoperative hospital stays. Among the trials, 2 constructed pairwise comparisons between $\mathrm{BI}$ and BII $(16,28), 4$ comparisons of BII and RY $(22,34,37,38), 17$ comparisons of BI and RY (7, 17-21, 24-27, 29-33, 35, 36), and 1 comparison of BII and URY were included (23). In addition, the only publication of the URY procedure was used to compare the remaining reconstruction skills.

In conclusion, there were 12 articles that assessed reflux gastritis, 8 articles on bile reflux, 14 on intraoperative blood loss and 15 on postoperative hospital stays in our study.

\section{Trial characteristics and study populations}

Table 1 shows the main baseline characteristics of the qualified trials in the network meta-analysis. Approximately 5419 patients were enrolled, and trials were mostly launched in Japan and Korea, except for 3 in China, 1 in France, 1 in the USA, 1 in Singapore, and 1 in Italy. There were 7 trials performed by at least 4 institutions, up to 18 institutions. Study dates varied from 1978 to 2016, and the average age varied from 56.3 to 71.5 years. BMI ranged from 22 to 26.2 , and the proportion of males ranged from $54.7 \%$ to $72.8 \%$. A network plot that depicts the interaction of the indirect comparisons is shown in Fig. 2.

\section{Methodological quality of the included studies}

The risk of bias identification of the included RCTs is presented in Fig. 3. Most of the studies were identified to have a low or unclear risk of bias for sequence generation and allocation concealment. Most studies were open label and were identified to have some unclear risks for the blinding of participants and personnel; however, once the authors 
reached an agreement that the performance bias would have few effects on the outcomes, we identified the study to have a low risk of bias. By contrast, a study would be at high risk if it was regarded to have an obvious influence on the outcomes. The same was true for the blinding of outcome assessments. In addition, almost all of the articles were identified to have a low risk of bias for incomplete outcome data and selective reporting, except for a minority of studies. The quality of non-RCTs was identified according to the NOS. As a result, all of the studies scored 7 to 9 points. The NOS assessment scale and results of each study are shown in Table 2 and Table 3 , respectively.

\section{Reflux gastritis}

Table 4 illustrates reflux gastritis among the reconstruction techniques. As shown in the table 4, the results in the gray region were generated from the researches with URY researches included, while the results in the green one was with the URY researches excluded. Our research showed that, compared to conventional BI reconstruction, RY appeared to have better strength in reducing reflux gastritis (OR 0.19,
95\%CI: 0.08 to 0.43 ) as well as compared to BII (OR $0.06,95 \% \mathrm{CI}: 0.01$ to 0.29$)$, but appeared to be no difference with URY (OR 8.19, 95\% CI: 0.48 to 133.91). What's more, URY showed to be no differences with BI and BII, either. The main reason may be that we only included one article containing URY which caused bias. On the other hand, when we excluded URY, RY still showed its superiority when compared with BI (OR 5.41, 95\%CI: 2.36 to 13.11) and BII (OR 16.78, 95\%CI: 3.59 to 77.44$)$. Besides, there were no differences among other comparisons.

\section{Bile reflux}

We expected that the RY technique might have an effective role in preventing bile reflux after gastrectomy. While Table 5 describes the unexpected result that a natural number of 0 was included in all confidence intervals whether URY was included or not, no significant differences were found in these comparisons. Generally, URY was expected to be the most likely to prevent reflux syndrome, but this failed to be the case. Thus, we did not find any differences between reconstruction skills in bile reflux.

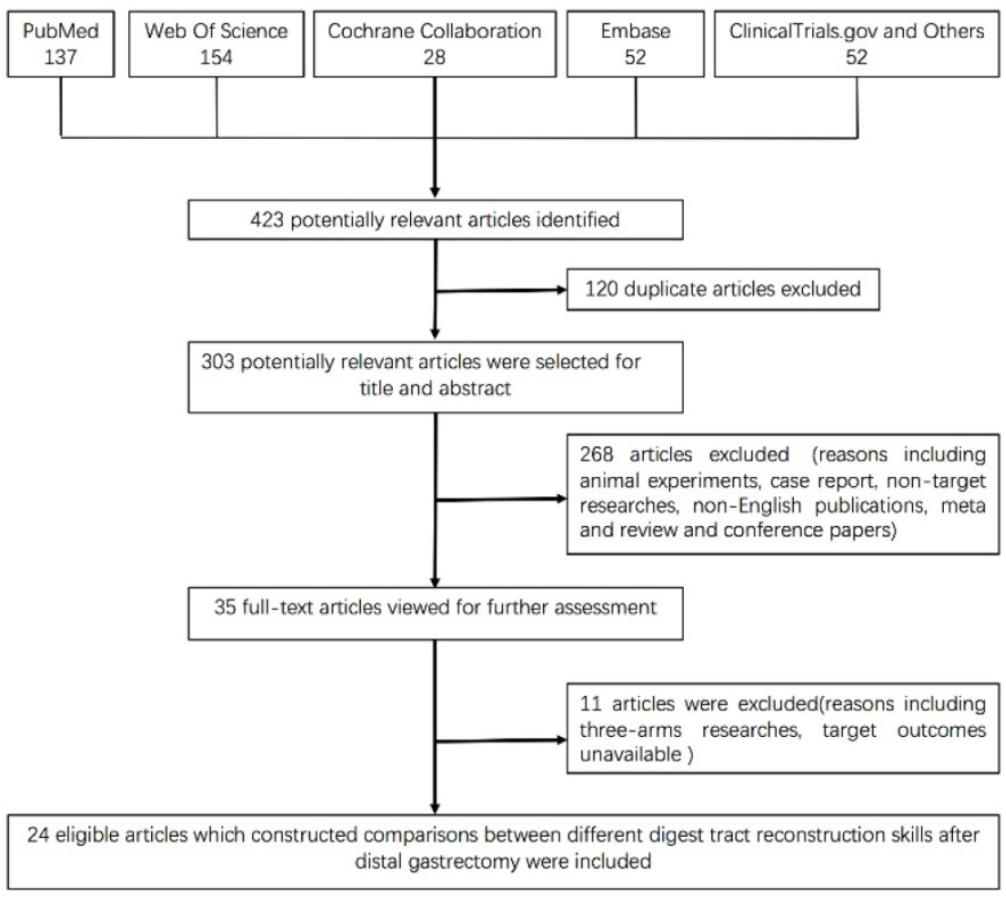

Figure 1. Flow chart of articles screening.

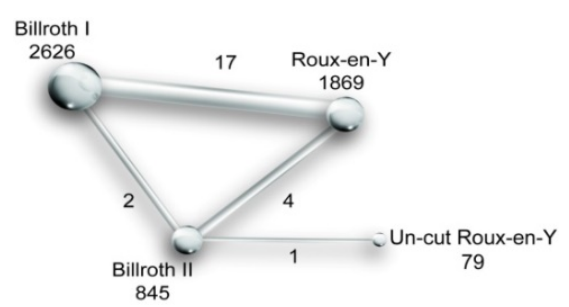

Figure 2. Network of indirect comparisons. The size of the nodes stands for the number of patients included and line width the number of articles comparing each pair of treatments. 
Table 1. Baseline characteristics and data extracted of clinical trials included in this study.

\begin{tabular}{|c|c|c|c|c|c|c|c|c|c|c|c|c|c|c|}
\hline Study & Design & Time & $\begin{array}{l}\text { Sources of } \\
\text { patients }\end{array}$ & $\begin{array}{l}\text { Reconst } \\
\text { ruction }\end{array}$ & Sex & TP & TN & $\mathrm{CN}$ & Age & BMI & $\begin{array}{l}\text { Postoperative } \\
\text { hospital stay }\end{array}$ & $\begin{array}{l}\text { Intraoperativ } \\
\text { e blood loss }\end{array}$ & $\begin{array}{l}\text { Reflux } \\
\text { gastritis }\end{array}$ & $\begin{array}{l}\text { Bile } \\
\text { reflux }\end{array}$ \\
\hline $\begin{array}{l}\text { Hiroshi } \\
\text { Imamura et } \\
\text { al. }\end{array}$ & $\begin{array}{l}\text { Randomized } \\
\text { Phase II } \\
\text { study }\end{array}$ & $\begin{array}{l}2005.08-20 \\
08.12\end{array}$ & $\begin{array}{l}18 \text { institutions } \\
\text { in Japan }\end{array}$ & $\mathrm{BI} / \mathrm{RY}$ & $\begin{array}{l}220 / 112 \\
(66.3 \%)\end{array}$ & 332 & 163 & 169 & $\begin{array}{l}64.4 \pm 9.3 / \\
63.9 \pm 10.5\end{array}$ & $\begin{array}{l}22.4 \pm 3 / \\
22.8 \pm 3.1\end{array}$ & $\begin{array}{l}14.1 \pm 6.5 / \\
16.4 \pm 10.4\end{array}$ & $\begin{array}{l}210 \pm 217 / \\
220 \pm 180\end{array}$ & NA & NA \\
\hline $\begin{array}{l}\text { Shuji } \\
\text { Takiguchi et } \\
\text { al. }\end{array}$ & $\begin{array}{l}\text { Randomized } \\
\text { Phase II } \\
\text { study }\end{array}$ & $\begin{array}{l}2005.08-20 \\
08.12\end{array}$ & $\begin{array}{l}18 \text { institutions } \\
\text { in Japan }\end{array}$ & $\mathrm{BI} / \mathrm{RY}$ & $\begin{array}{l}178 / 90 \\
(66.4 \%)\end{array}$ & 268 & 132 & 136 & $\begin{array}{l}64.5 \pm 9.8 / \\
64.1 \pm 10.5\end{array}$ & NA & NA & $\begin{array}{l}210 \pm 230 / \\
203 \pm 153\end{array}$ & NA & NA \\
\hline $\begin{array}{l}\text { Bruno } \\
\text { Chareton et } \\
\text { al. }\end{array}$ & $\mathrm{RCT}$ & $\begin{array}{l}1978.01-19 \\
89.04\end{array}$ & $\begin{array}{l}\text { one institution } \\
\text { in France }\end{array}$ & $\mathrm{BI} / \mathrm{BII}$ & $\begin{array}{l}41 / 19 \\
(66.1 \%)\end{array}$ & 62 & 30 & 32 & $\begin{array}{l}71.5 \pm 8.5 / \\
70.6 \pm 8.4\end{array}$ & NA & $\begin{array}{l}16.9 \pm 11.9 / \\
13 \pm 4.8\end{array}$ & NA & NA & NA \\
\hline $\begin{array}{l}\text { Makoto } \\
\text { Ishikawa et } \\
\text { al. }\end{array}$ & RCT & $\begin{array}{l}2001.01-20 \\
04.09\end{array}$ & $\begin{array}{l}\text { one institution } \\
\text { in Tokyo, Japan }\end{array}$ & $\mathrm{BI} / \mathrm{RY}$ & $\begin{array}{l}36 / 14 \\
(72.0 \%)\end{array}$ & 50 & 26 & 24 & $\begin{array}{l}64(34-84) / \\
64(43-80)\end{array}$ & NA & $19 \pm 6 / 31.8 \pm 21.7$ & $\begin{array}{l}374 \pm 392 / \\
32 \pm 250\end{array}$ & $\begin{array}{l}(16 / 26) /(7 \\
/ 23)\end{array}$ & $\begin{array}{l}(8 / 26) /( \\
0 / 23)\end{array}$ \\
\hline $\begin{array}{l}\text { Moon-Soo } \\
\text { Lee }\end{array}$ & RCT & $\begin{array}{l}2006.03-20 \\
07.08\end{array}$ & $\begin{array}{l}\text { one institution } \\
\text { in Seoul }\end{array}$ & $\mathrm{BI} / \mathrm{RY}$ & $\begin{array}{l}59 / 37 \\
(61.5 \%)\end{array}$ & 96 & 49 & 47 & $\begin{array}{l}60.6 \pm 11.6 / \\
58.5 \pm 10.7\end{array}$ & NA & $\begin{array}{l}9.2 \pm 3.1 / \\
10.8 \pm 7.7\end{array}$ & NA & NA & NA \\
\hline $\begin{array}{l}\text { M.Nakamura } \\
\text { et al. }\end{array}$ & RCT & $\begin{array}{l}2009.01-20 \\
10.09\end{array}$ & $\begin{array}{l}\text { multicenter in } \\
\text { Japan }\end{array}$ & $\mathrm{BI} / \mathrm{RY}$ & $\begin{array}{l}85 / 37 \\
(69.7 \%)\end{array}$ & 122 & 60 & 62 & $\begin{array}{l}66(40-80) / \\
67(43-80)\end{array}$ & NA & $\begin{array}{l}11 \pm 14 / \\
11 \pm 20.25\end{array}$ & $\begin{array}{l}153 \pm 181.1 / \\
221.4 \pm 239\end{array}$ & $\begin{array}{l}(35 / 53) /(1 \\
5 / 52)\end{array}$ & $\begin{array}{l}(39 / 53) \\
/(52 / 52 \\
)\end{array}$ \\
\hline $\begin{array}{l}\text { Yoon Young } \\
\text { Choi et al. }\end{array}$ & RCT & $\begin{array}{l}2011.07-20 \\
14-05\end{array}$ & $\begin{array}{l}\text { one institution } \\
\text { in Korea }\end{array}$ & $\mathrm{BI} / \mathrm{RY}$ & $\begin{array}{l}26 / 14 \\
(65.0 \%)\end{array}$ & 40 & 20 & 20 & $\begin{array}{l}62.4 \pm 8.9 / \\
62.8 \pm 9.3\end{array}$ & NA & $6.7 \pm 1 / 7.1 \pm 1.1$ & $\begin{array}{l}93.1 \pm 54.5 / \\
69.9 \pm 53.4\end{array}$ & NA & NA \\
\hline $\begin{array}{l}\text { Jimmy } \\
\text { Bok-Yan So et } \\
\text { al. }\end{array}$ & RCT & $\begin{array}{l}2008.10-20 \\
14.10\end{array}$ & $\begin{array}{l}4 \text { institution in } \\
\text { Singapore and } \\
\text { Hong Kong }\end{array}$ & BII/RY & $\begin{array}{l}91 / 71 \\
(56.2 \%)\end{array}$ & 162 & 81 & 81 & $\begin{array}{l}62 \pm 10.9 / \\
64.5 \pm 10.9\end{array}$ & $\begin{array}{l}23.3 \pm 4.1 \\
/ 24.3 \pm 4 . \\
2\end{array}$ & $\begin{array}{l}9 \pm 0.833 / \\
8 \pm 0.667\end{array}$ & NA & $\begin{array}{l}(31 / 33) /(1 \\
7 / 51)\end{array}$ & NA \\
\hline $\begin{array}{l}\text { Dong Yang et } \\
\text { al. }\end{array}$ & RCT & $\begin{array}{l}2015.02-20 \\
16.02\end{array}$ & $\begin{array}{l}\text { one institution } \\
\text { in China }\end{array}$ & $\begin{array}{l}\text { BII/UR } \\
\text { Y }\end{array}$ & $\begin{array}{l}114 / 44 \\
(72.2 \%)\end{array}$ & 158 & 79 & 79 & $\begin{array}{l}61.8 \pm 11.4 / \\
58 \pm 11.4\end{array}$ & NA & NA & $\begin{array}{l}74 \pm 36.6 / \\
74.1 \pm 26.7\end{array}$ & $\begin{array}{l}(52 / 72) /(3 \\
8 / 69)\end{array}$ & $\begin{array}{l}(65 / 72) \\
/(42 / 69 \\
)\end{array}$ \\
\hline $\begin{array}{l}\text { Kun Yang et } \\
\text { al. }\end{array}$ & RCT & $\begin{array}{l}2011.05-20 \\
14.05\end{array}$ & $\begin{array}{l}\text { one institution } \\
\text { in China }\end{array}$ & $\mathrm{BI} / \mathrm{RY}$ & $\begin{array}{l}87 / 53 \\
(62.1 \%)\end{array}$ & 140 & 70 & 70 & $\begin{array}{l}56.3 \pm 10.7 / \\
54.9 \pm 11.5\end{array}$ & $\begin{array}{l}22.4 \pm 3.1 \\
/ 22.7 \pm 3 \\
5\end{array}$ & $\begin{array}{l}9.6 \pm 1.2 / \\
10.3 \pm 3.7\end{array}$ & $\begin{array}{l}84.1 \pm 32 / \\
104.2 \pm 35.3\end{array}$ & $\begin{array}{l}(38 / 46) /(2 \\
7 / 46)\end{array}$ & $\begin{array}{l}(9 / 46) /( \\
4 / 46)\end{array}$ \\
\hline $\begin{array}{l}\text { Souya } \\
\text { Nunobe et al. }\end{array}$ & $\begin{array}{l}\text { Retrospective } \\
\text { Study }\end{array}$ & $\begin{array}{l}1993.01-19 \\
99.12\end{array}$ & $\begin{array}{l}\text { one institution } \\
\text { in Japan }\end{array}$ & $\mathrm{BI} / \mathrm{RY}$ & $\begin{array}{l}224 / 141 \\
(63.4 \%)\end{array}$ & 443 & 229 & 214 & NA & NA & NA & NA & $\begin{array}{l}(17 / 203) / \\
(3 / 188)\end{array}$ & $\begin{array}{l}(17 / 203 \\
) /(9 / 18 \\
2)\end{array}$ \\
\hline $\begin{array}{l}\text { Kazuyuzi } \\
\text { Kojima et al. }\end{array}$ & $\begin{array}{l}\text { Retrospective } \\
\text { Study }\end{array}$ & $\begin{array}{l}2000.10-20 \\
06.02\end{array}$ & $\begin{array}{l}\text { one institution } \\
\text { in Japan }\end{array}$ & $\mathrm{BI} / \mathrm{RY}$ & $\begin{array}{l}91 / 42 \\
(68.4 \%)\end{array}$ & 133 & 65 & 68 & $\begin{array}{l}62 \pm 8.9 / 62 \\
8 \pm 12.2\end{array}$ & $\begin{array}{l}22 \pm 2.5 / \\
23 \pm 3\end{array}$ & $12.4 \pm 4 / 7 \pm 2$ & $\begin{array}{l}103.6 \pm 97.1 / \\
80.5 \pm 95.2\end{array}$ & $\begin{array}{l}(22 / 65) / \\
(8 / 68)\end{array}$ & $\begin{array}{l}(21 / 65) \\
/(0 / 68)\end{array}$ \\
\hline $\begin{array}{l}\text { Tsutomo } \\
\text { Namikawa et } \\
\text { al. }\end{array}$ & $\begin{array}{l}\text { Retrospective } \\
\text { Study }\end{array}$ & 2005-2008 & $\begin{array}{l}\text { one institution } \\
\text { in Japan }\end{array}$ & $\mathrm{BI} / \mathrm{RY}$ & $\begin{array}{l}47 / 38(5 \\
5.3 \%)\end{array}$ & 85 & 47 & 38 & $\begin{array}{l}70.5 \pm 10.9 / \\
66.2 \pm 11.4\end{array}$ & NA & NA & NA & NA & NA \\
\hline $\begin{array}{l}\text { Kyu-Chul } \\
\text { Kang et al. }\end{array}$ & $\begin{array}{l}\text { Retrospective } \\
\text { Study }\end{array}$ & $\begin{array}{l}1998.04-20 \\
05.12\end{array}$ & $\begin{array}{l}10 \text { institution in } \\
\text { Japan }\end{array}$ & $\mathrm{BI} / \mathrm{BII}$ & $\begin{array}{l}792 / 467 \\
(62.9 \%)\end{array}$ & $\begin{array}{l}125 \\
9\end{array}$ & 875 & 384 & $\begin{array}{l}58.0 \pm 12.1 / \\
57.5 \pm 12.1\end{array}$ & NA & $\begin{array}{l}9.1 \pm 17.5 / \\
10.6 \pm 8.9\end{array}$ & NA & NA & NA \\
\hline $\begin{array}{l}\text { Koshi } \\
\text { Kumagai et } \\
\text { al. }\end{array}$ & $\begin{array}{l}\text { Retrospective } \\
\text { Study }\end{array}$ & $\begin{array}{l}2005.04-20 \\
09.07\end{array}$ & $\begin{array}{l}\text { one institution } \\
\text { in Japan }\end{array}$ & $\mathrm{BI} / \mathrm{RY}$ & $\begin{array}{l}271 / 153 \\
(63.9 \%)\end{array}$ & 424 & 329 & 95 & $\begin{array}{l}63.5(29-90) \\
/ 62.7(42-8 \\
1)\end{array}$ & $\begin{array}{l}23.2 \pm 3.5 \\
/ 24 \pm 3.3\end{array}$ & $\begin{array}{l}12.6 \pm 6.4 / \\
14 \pm 9.5\end{array}$ & $\begin{array}{l}62 \pm 142 / \\
84 \pm 127\end{array}$ & NA & NA \\
\hline $\begin{array}{l}\text { Shinnosuke } \\
\text { Tanaka et al. }\end{array}$ & $\begin{array}{l}\text { Retrospective } \\
\text { Study }\end{array}$ & $\begin{array}{l}1998.01-20 \\
06.09\end{array}$ & $\begin{array}{l}\text { one institution } \\
\text { in Japan }\end{array}$ & $\mathrm{BI} / \mathrm{RY}$ & $\begin{array}{l}68 / 33(6 \\
7.3 \%)\end{array}$ & 101 & 50 & 51 & $66.2 / 65.2$ & NA & NA & NA & $\begin{array}{l}(24 / 35) /(7 \\
/ 31)\end{array}$ & NA \\
\hline $\begin{array}{l}\text { Sang-Woong } \\
\text { Lee et al. }\end{array}$ & $\begin{array}{l}\text { Retrospective } \\
\text { Study }\end{array}$ & $\begin{array}{l}2000.06-20 \\
11.12\end{array}$ & $\begin{array}{l}\text { one institution } \\
\text { in Japan }\end{array}$ & $\mathrm{BI} / \mathrm{RY}$ & $\begin{array}{l}237 / 139 \\
(63.0 \%)\end{array}$ & 376 & 248 & 128 & $\begin{array}{l}66 \pm 11 / \\
66 \pm 10\end{array}$ & NA & NA & $47 \pm 48 / 76 \pm 80$ & NA & NA \\
\hline $\begin{array}{l}\text { Mikito } \\
\text { Inokuchi }\end{array}$ & $\begin{array}{l}\text { Retrospective } \\
\text { Study }\end{array}$ & $\begin{array}{l}1999.01-20 \\
06.08\end{array}$ & $\begin{array}{l}\text { one institution } \\
\text { in Japan }\end{array}$ & $\mathrm{BI} / \mathrm{RY}$ & $\begin{array}{l}113 / 59( \\
65.7 \%)\end{array}$ & 172 & 89 & 83 & 62 & $\begin{array}{l}22 \pm 2.5 / \\
23.1 \pm 3.2\end{array}$ & NA & NA & $\begin{array}{l}(75 / 80) / \\
(27 / 80)\end{array}$ & $\begin{array}{l}(43 / 80) \\
/(7 / 80)\end{array}$ \\
\hline $\begin{array}{l}\text { Ji Yeong An } \\
\text { et al. }\end{array}$ & $\begin{array}{l}\text { Retrospective } \\
\text { Study }\end{array}$ & $\begin{array}{l}2011.01-20 \\
12.05\end{array}$ & $\begin{array}{l}\text { one institution } \\
\text { in Korea }\end{array}$ & $\mathrm{BI} / \mathrm{RY}$ & $\begin{array}{l}58 / 42 \\
(58.0 \%)\end{array}$ & 100 & 50 & 50 & $\begin{array}{l}58.2 \pm 13.2 / \\
59 \pm 11.9\end{array}$ & $\begin{array}{l}23.3 \pm 2.9 \\
/ 25.2 \pm 3\end{array}$ & $7.2 \pm 1.2 / 7.2 \pm 1$ & $\begin{array}{l}54.2 \pm 34 / \\
68.4 \pm 38.9\end{array}$ & NA & NA \\
\hline $\begin{array}{l}\text { Jung Ho Shim } \\
\text { et al. }\end{array}$ & $\begin{array}{l}\text { Retrospective } \\
\text { Study }\end{array}$ & $\begin{array}{l}2011.01-20 \\
11.06\end{array}$ & $\begin{array}{l}\text { one institution } \\
\text { in Korea }\end{array}$ & $\mathrm{BII} / \mathrm{RY}$ & $\begin{array}{l}59 / 22 \\
(72.8 \%)\end{array}$ & 81 & 43 & 38 & $\begin{array}{l}58(33-78) / \\
60.9(31-79)\end{array}$ & $\begin{array}{l}24 \pm 3.93 \\
/ 26 \pm 2.7 \\
7\end{array}$ & $6.8 \pm 2.6 / 8.2 \pm 3.4$ & NA & $\begin{array}{l}(30 / 33) / \\
(5 / 27)\end{array}$ & $\begin{array}{l}(29 / 33) \\
/(2 / 27)\end{array}$ \\
\hline $\begin{array}{l}\text { Shuhei } \\
\text { Komatsu et } \\
\text { al. }\end{array}$ & $\begin{array}{l}\text { Retrospective } \\
\text { Study }\end{array}$ & 2007-2010 & $\begin{array}{l}\text { one institution } \\
\text { in Japan }\end{array}$ & $\mathrm{BI} / \mathrm{RY}$ & $\begin{array}{l}64 / 53 \\
(54.7 \%)\end{array}$ & 117 & 74 & 43 & $61.9 / 65.2$ & NA & NA & NA & NA & NA \\
\hline $\begin{array}{l}\text { Taisuke } \\
\text { Imamura et } \\
\text { al. }\end{array}$ & $\begin{array}{l}\text { Retrospective } \\
\text { Study }\end{array}$ & $\begin{array}{l}2006.01-20 \\
12.04\end{array}$ & $\begin{array}{l}\text { one institution } \\
\text { in Japan }\end{array}$ & $\mathrm{BI} / \mathrm{RY}$ & $\begin{array}{l}74 / 45 \\
(62.2 \%)\end{array}$ & 119 & 77 & 42 & $\begin{array}{l}59.7 \pm 1.3 / \\
64.9 \pm 1.7\end{array}$ & $\begin{array}{l}22.41 \pm 0 \\
38 / 22.8 \\
\pm 0.54\end{array}$ & NA & $\begin{array}{l}93 \pm 17.9 / 184 \\
2 \pm 24.3\end{array}$ & NA & NA \\
\hline $\begin{array}{l}\text { Thuy B. Tran } \\
\text { et al. }\end{array}$ & $\begin{array}{l}\text { Retrospective } \\
\text { Cohort Study }\end{array}$ & 2000-2012 & $\begin{array}{l}7 \text { institution in } \\
\text { the USA }\end{array}$ & $\mathrm{BII} / \mathrm{RY}$ & $\begin{array}{l}249 / 198 \\
(55.7 \%)\end{array}$ & 447 & 190 & 257 & $\begin{array}{l}67 \pm 12 / \\
65 \pm 13\end{array}$ & $\begin{array}{l}25.9 \pm 6.4 \\
/ 26.2 \pm 5 . \\
6\end{array}$ & $\begin{array}{l}7 \pm 0.833 / \\
8 \pm 0.667\end{array}$ & $\begin{array}{l}205 \pm 151 / 243 \\
\pm 195\end{array}$ & NA & NA \\
\hline $\begin{array}{l}\text { Edoardo } \\
\text { Virgilio et al. }\end{array}$ & $\begin{array}{l}\text { Retrospective } \\
\text { Study }\end{array}$ & $\begin{array}{l}2005.04-20 \\
16.02\end{array}$ & $\begin{array}{l}5 \text { institution in } \\
\text { the Italy }\end{array}$ & $\mathrm{BII} / \mathrm{RY}$ & $\begin{array}{l}76 / 56 \\
(57.6 \%)\end{array}$ & 132 & 36 & 96 & $\begin{array}{l}67 \pm 10 / \\
68 \pm 13\end{array}$ & NA & $\begin{array}{l}16.6 \pm 23 / \\
13.6 \pm 11\end{array}$ & $\begin{array}{l}186 \pm 148 / 157 \\
\pm 116\end{array}$ & $\begin{array}{l}(3 / 36) / \\
(4 / 100)\end{array}$ & NA \\
\hline
\end{tabular}

\section{Intraoperative blood loss}

Surgical safety was an essential aspect that surgeons considered; however, no significant difference was found in the comparisons, as listed in Table 6. However, we observed that the confidence intervals had a large scope, which indicated the possible existence of some extreme values.

\section{Postoperative hospital stays}

Only three reconstruction skills were analyzed in this study due to postoperative hospital stays not being a target of interest in the article that referred to the URY technique. Table 7 suggests there were no differences in the indirect pairwise comparisons. In addition, a node split, which tested the consistency of 

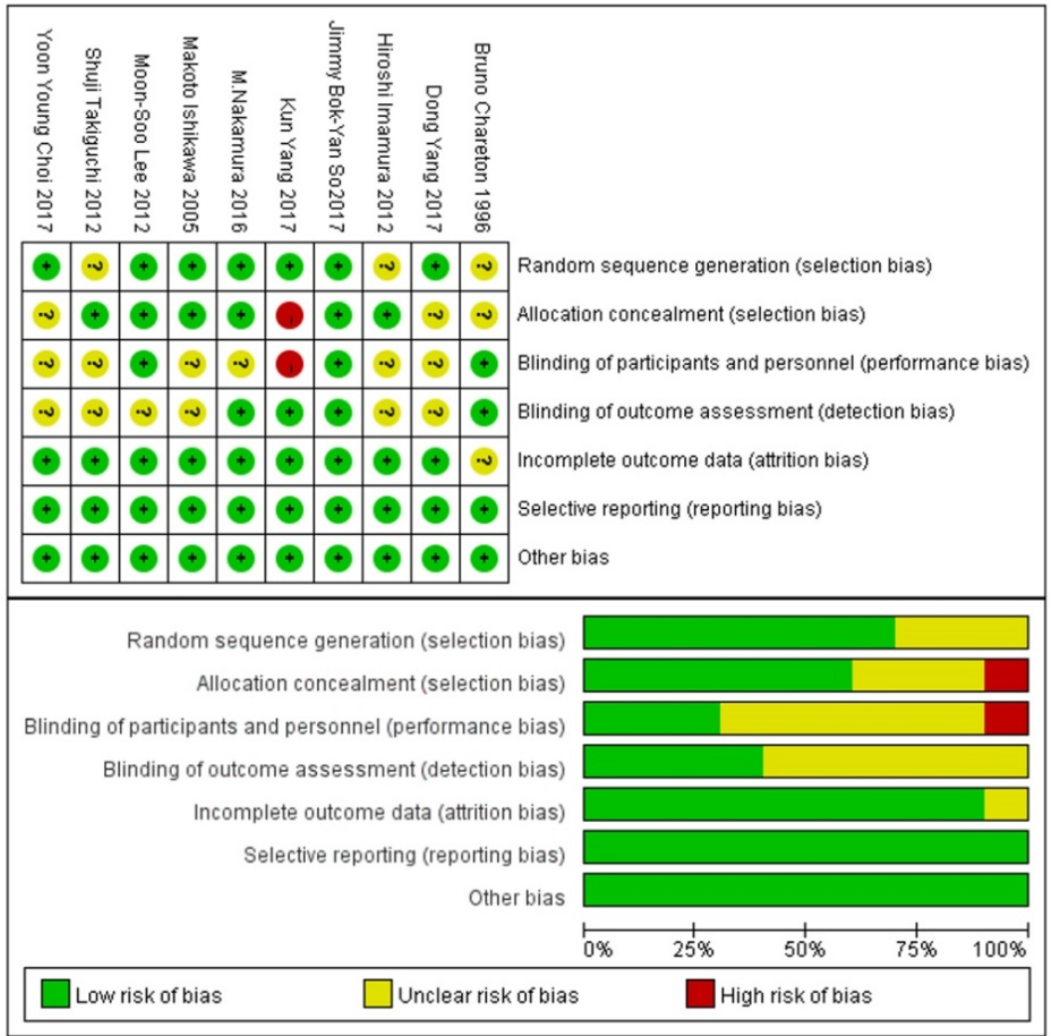

Figure 3. Risk of bias graph and risk of bias summary of RCTs

direct and indirect comparisons, revealed a good fit, with a $\mathrm{p}>0.05$.

Table 2. Newcastle-Ottawa quality assessment scale*

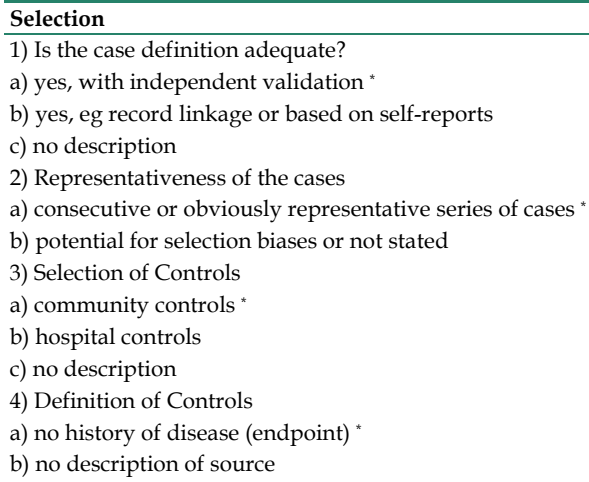

\section{Comparability}

1) Comparability of cases and controls on the basis of the design or analysis a) study controls for "age, sex, Body Mass Index" (Select the most important factor.) *

b) study controls for any additional factor * (This criteria could be modified to indicate specific control for a second important factor.)

\section{Exposure}

1) Ascertainment of exposure

a) secure record (eg surgical records) *

b) structured interview where blind to case/control status *

c) interview not blinded to case/control status

d) written self-report or medical record only

e) no description

2) Same method of ascertainment for cases and controls a) yes * b) no

3) Non-Response rate

a) same rate for both groups *

b) non respondents described c) rate different and no designation

*A study can be awarded a maximum of one star for each numbered item within the Selection and Exposure categories. A maximum of two stars can be given for Comparability.

Table 3. Assessment of Quality of Studies

\begin{tabular}{|c|c|c|c|c|c|c|c|c|c|}
\hline \multirow[b]{2}{*}{ References } & \multicolumn{4}{|c|}{ selection } & \multirow{2}{*}{$\begin{array}{l}\text { comparability } \\
5\end{array}$} & \multicolumn{4}{|c|}{ outcome } \\
\hline & 1 & 2 & 3 & 4 & & 6 & 7 & 8 & score \\
\hline 2011 Kumagai et al. & $b^{*}$ & $a^{*}$ & $a^{*}$ & $a^{*}$ & $a b^{* *}$ & $a^{*}$ & $a^{*}$ & $a^{*}$ & 9 \\
\hline 2013 Inokuchi et al. & $b^{*}$ & $a^{*}$ & $a^{*}$ & $a^{*}$ & $a^{*}$ & $a^{*}$ & $a^{*}$ & c & 7 \\
\hline 2007 Nunobe et al. & $b^{*}$ & $a^{*}$ & $a^{*}$ & $a^{*}$ & $b^{*}$ & $a^{*}$ & $a^{*}$ & $a^{*}$ & 8 \\
\hline 2008 Kojima et al. & $b^{*}$ & $a^{*}$ & $a^{*}$ & $a^{*}$ & $a b * *$ & $a^{*}$ & $a^{*}$ & $a^{*}$ & 9 \\
\hline 2010 Namikawa et al. & $b^{*}$ & $a^{*}$ & $a^{*}$ & $a^{*}$ & $a b^{*}$ & $a^{*}$ & $a^{*}$ & $a^{*}$ & 8 \\
\hline 2011 Kang et al. & $b^{*}$ & $a^{*}$ & $a^{*}$ & $a^{*}$ & $a^{*}$ & $a^{*}$ & $a^{*}$ & c & 7 \\
\hline 2011 Tanaka et al. & $b^{*}$ & $a^{*}$ & $a^{*}$ & $a^{*}$ & $a b^{*}$ & $a^{*}$ & $a^{*}$ & c & 7 \\
\hline 2012 Lee et al. & $b^{*}$ & $a^{*}$ & $a^{*}$ & $a^{*}$ & $a b^{* *}$ & $a^{*}$ & $a^{*}$ & $a^{*}$ & 9 \\
\hline 2014 An et al. & $b^{*}$ & $a^{*}$ & $a^{*}$ & $a^{*}$ & $a^{*}$ & $a^{*}$ & $a^{*}$ & $a^{*}$ & 8 \\
\hline 2014 Shim et al. & $b^{*}$ & $a^{*}$ & $a^{*}$ & $a^{*}$ & $a *$ & $a^{*}$ & $a^{*}$ & $a^{*}$ & 8 \\
\hline 2015 Komatsu et al. & $b^{*}$ & $a^{*}$ & $a^{*}$ & $a^{*}$ & $b^{*}$ & $a^{*}$ & $a^{*}$ & $a^{*}$ & 8 \\
\hline 2016 Imamura et al. & $b^{*}$ & $a^{*}$ & $a^{*}$ & $a^{*}$ & $a b^{* *}$ & $a^{*}$ & $a^{*}$ & c & 8 \\
\hline 2016 Tran et al. & $b^{*}$ & $a^{*}$ & $a^{*}$ & $a^{*}$ & $a b^{* *}$ & $a^{*}$ & $a^{*}$ & $a^{*}$ & 9 \\
\hline 2017 Edoardo et al. & $b^{*}$ & $\mathrm{~b}$ & $a^{*}$ & $a^{*}$ & $a b^{*}$ & $a^{*}$ & $a^{*}$ & $a^{*}$ & 7 \\
\hline
\end{tabular}

\section{Ranking of the different digestive tract reconstruction techniques}

Network meta-analysis also offered a comprehensive ranking when exploring the reconstruction that was the most likely to contribute in corresponding 
areas using corresponding SUCRA values. Fig. 4 shows that RY worked best at preventing reflux gastritis, followed by BI, URY, and BII. Almost the same results were found with respect to bile reflux, except for the interchange of the position of BI and URY. On the other hand, URY was more likely to bleed more during the surgical procedure, which made BI appear to be more reliable regarding safety. Ultimately, RY appeared to have a relatively high ranking in postoperative hospital stays, as suggested by the SUCRA value. This result matched the reflux syndromes, indicating a possible relation between them.

Table 4. Comparison of different reconstruction in reflux gastritis between pairwise meta-analysis and network.

\begin{tabular}{llll}
\hline BI & $3.14(0.50,18.84)$ & $0.19(0.08,0.43)$ & $1.51(0.08,26.82)$ \\
$0.32(0.06,1.92)$ & BII & $0.06(0.01,0.29)$ & $0.48(0.05,5.07)$ \\
$5.41(2.36,13.11)$ & $16.78(3.59,77.44)$ & RY & $8.19(0.48,133.91)$ \\
& & & URY \\
\hline
\end{tabular}

BI: Billroth I reconstruction; BII: Billroth II reconstruction; RY: Roux-en-Y reconstruction; URY: Un-cut Roux-en-Y. Results were expressed using odds ratio and $95 \%$ confidence interval. The column reconstruction was compared with the row construction.

Table 5. Comparison of different reconstruction in bile reflux between pairwise meta-analysis and network.

\begin{tabular}{llll}
\hline BI & 23.35 & 0.18 & 3.35 \\
& $(0.01,51378.37)$ & $(0.01,3.35)$ & $(0.00,105142.49)$ \\
0.04 & BII & 0.01 & 0.16 \\
$(0.00,91.69)$ & & $(0.00,10.12)$ & $(0.00,139.34)$ \\
5.59 & 129.55 & RY & 20.29 \\
$(0.28,143.111)$ & $(0.13,137248.80)$ & & $(0.00,381143.45)$ \\
& & & URY \\
\hline
\end{tabular}

BI: Billroth I reconstruction; BII: Billroth II reconstruction; RY: Roux-en-Y reconstruction; URY: Un-cut Roux-en-Y. Results were expressed using odds ratio and $95 \%$ confidence interval. The column reconstruction was compared with the row construction.

Table 6. Comparison of different reconstruction in intraoperative blood loss between pairwise meta-analysis and network.

\begin{tabular}{llll}
\hline BI & 375.72 & 377.16 & 366.71 \\
& $(-1695.01,2371.29)$ & $(-443.92,1188.77)$ & $(-3002.17,3677.56)$ \\
-376.53 & BII & -10.00 & -0.92 \\
$(-2425.31,1673.35)$ & & $(-1861.31,1906.65)$ & $(-2675.22,2641.25)$ \\
-383.21 & -7.03 & RY & -9.34 \\
$(-1196.92,430.09)$ & $(-1887.59,1870.03)$ & & $(-3291.99,3208.19)$ \\
& & & URY \\
\hline
\end{tabular}

BI: Billroth I reconstruction; BII: Billroth II reconstruction; RY: Roux-en-Y reconstruction; URY: Un-cut Roux-en-Y. Results were expressed using odds ratio and $95 \%$ confidence interval. The column reconstruction was compared with the row construction.

Table 7. Comparison of different reconstruction in post-operative hospital stay between pairwise meta-analysis and network.

\begin{tabular}{lll}
\hline BI & $-0.03(-2.79,2.79)$ & $0.35(-1.48,2.41)$ \\
$0.03(-2.97,2.97)$ & BII & $0.38(-2.19,3.11)$ \\
$-0.35-2.41,1.48)$ & $-0.38(-3.11,2.19)$ & RY \\
\hline BI: Billroth I reconstruction; BII: Billroth II reconstruction; RY: Roux-en-Y \\
reconstruction. Results were expressed using odds ratio and 95\% confidence \\
interval. The column reconstruction was compared with the row construction.
\end{tabular}

\section{Discussion}

This indirect comparison of different reconstruction methods after radical distal gastrectomy across 10 RCTs and 14 retrospective clinical trials (5419 individuals) suggested that RY has the highest probability at achieving reflux gastritis reduction without increasing the postoperative hospital stay. We believe that patients are more willing to choose RY reconstruction for the purpose of removing the displeasure caused by reflux symptoms, despite no significant difference being estimated for the reduction of bile reflux. In addition, as a newly emerging technique, URY was deemed to lead to more intraoperative blood loss, but did not offer a benefit in reflux symptom control. In addition, there was no significant difference found in hospitalization stay between BI, BII and RY. The results of the analysis, except for URY, were consistent with the aforementioned results.

$\mathrm{BI}$ is still the standard reconstruction in some Western countries because of its physiological rationality, superiority in terms of the quick recovery of the nutritional status as well as the simplicity of the surgical procedure. The SUCRA value indicated a $44 \%$ probability of BI providing the greatest blood loss benefit, and it was equivalent to or even better than BII regarding the reduction of reflux symptoms. These results may be explained by the absence of the pylorus. On the other hand, some investigators have proposed that the angle of HIS was of vital importance in the reflux mechanism (27).

Technically, URY builds a bridge that maintains neuromuscular continuity between the proximal jejunum and the Roux Limb instead of cutting it off. URY was initially expected by Stiegman and Goff to release reflux symptoms and Roux stasis (14), but it was left to be statistically identified in our indirect comparison. Despite this, URY still showed a trend of greater superiority than BII in terms of reflux gastritis and bile reflux in our study, which was imaginable because URY itself was a technical improvement based on BII reconstruction. Besides, Park's study also suggested that, compared to RY, URY had more evidence for managing reflux and decreasing anastomotic leakage simultaneously (39). It may be related to Braun anastomosis, which alleviates the pressure of the afferent loop. This result contradicts our study. Some reasons for this conflict include, first, only one URY related article with a small number was included in our analysis, which may have resulted in bias. Second, the article identified a partial recanalization rate of $13 \%$ of un-cut closures, contributing to $60.9 \%$ of the bile reflux rate. Generally, with the recanalization of the un-cut closure, URY is somewhat equivalent to RY reconstruction, despite 
the Braun anastomosis. Therefore, our study did not reach our expectation, and our outcomes should be interpreted with caution.

Meta-analysis of reconstruction techniques has been previously used to study special reconstructions. Zong and Chen reached the same results as we did, except they found a shorter hospital stay in the RY group (3). Jun-Jie Xiong et al. (4) clarified that RY had the effect of reducing reflux esophagitis, reflux gastritis and bile reflux, which was mostly consistent with our study. Different from Zong's study, 3-arm studies were excluded in our study because we
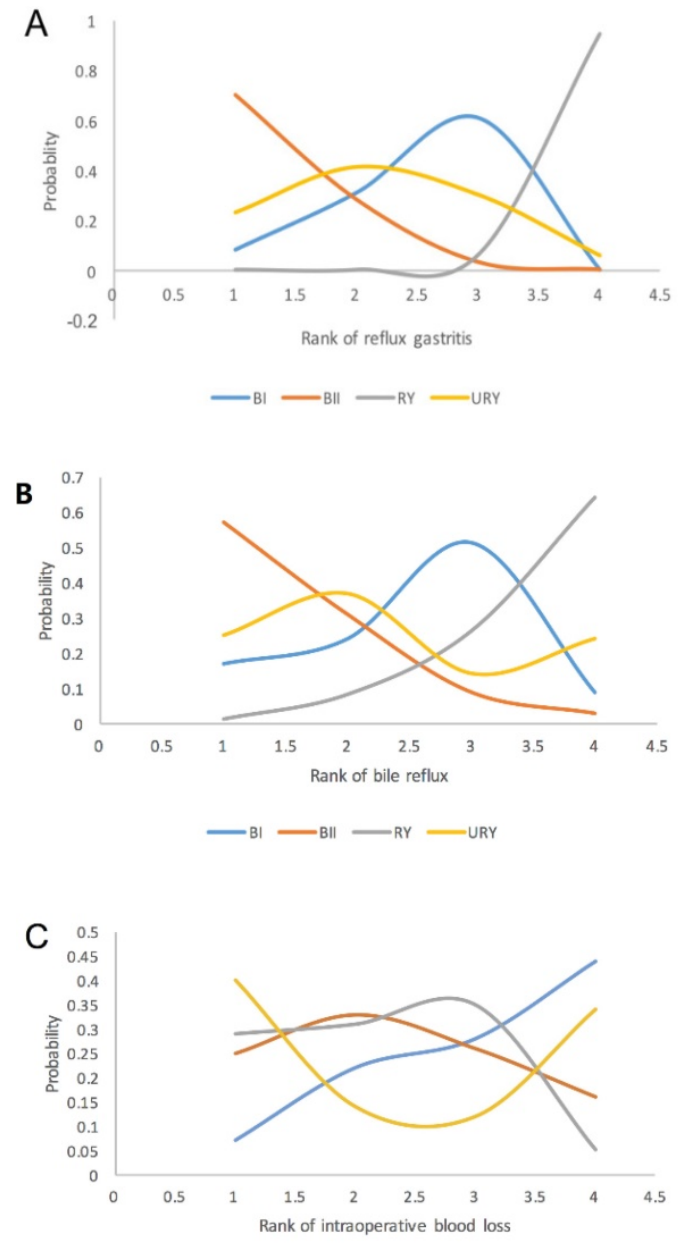

$-\mathrm{BI}-\mathrm{BII}-\mathrm{RY}-\mathrm{URY}$

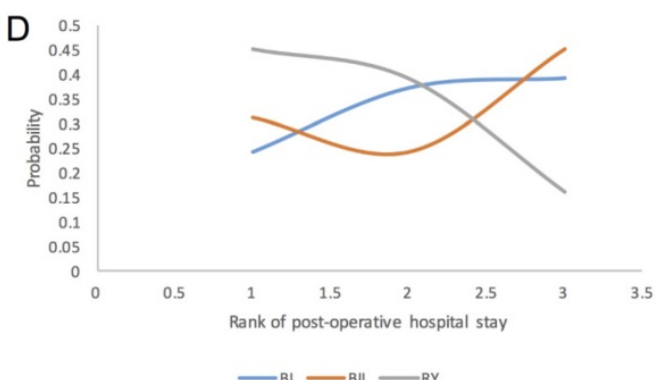

believed that they may cause statistical deviations. Besides, we are the first to include URY, a technique expected as the future surgical procedure to solve severe reflux symptoms for distal gastric cancer, and the result showed certain advantages in postoperative complications. Furthermore, we performed an indirect comparison that was considered to be a surrogate for a head-to-head treatment comparison to further confirm our results. We obtained good consistency between direct comparisons and indirect comparisons.
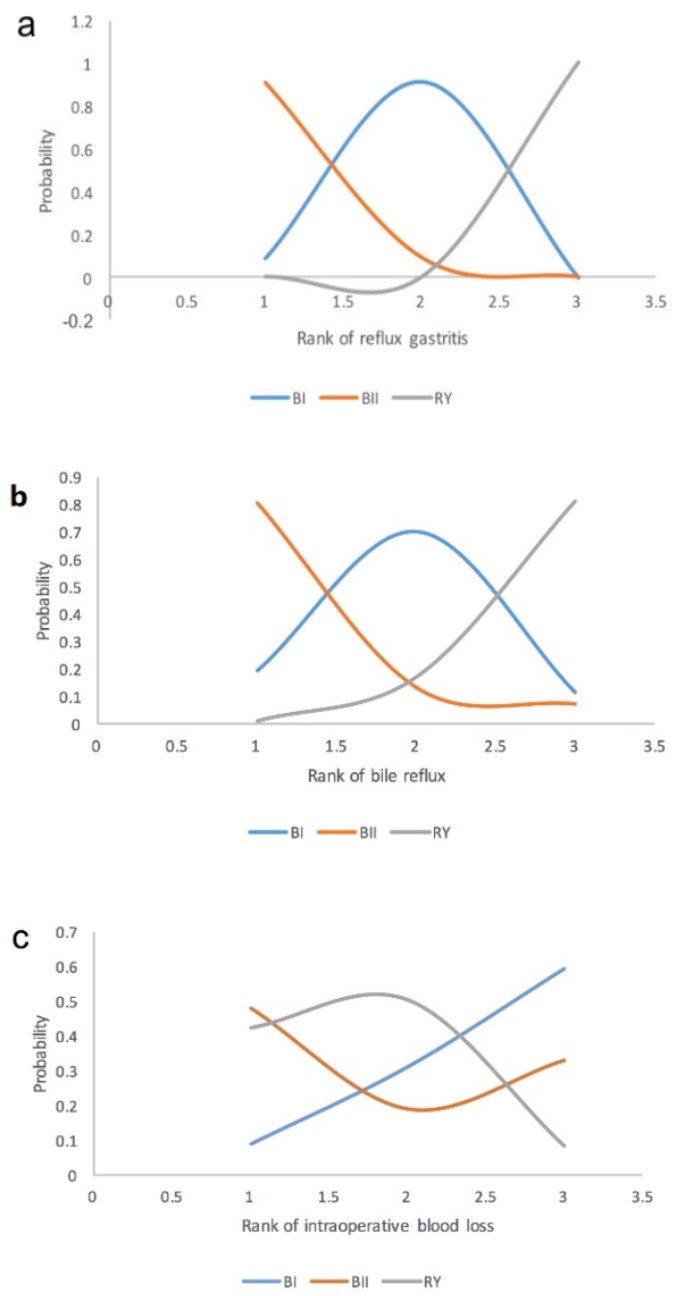

Figure 4. Ranking of outcomes for the included studies. Odds ratio(OR) and $95 \%$ confidence interval(Cl) were used to expressed the difference between reconstructions. First rank indicates lowest probability to prevent occurrence of reflux gastritis and bile reflux. For intraoperative blood loss, first rank indicates highest probability of more blood loss. For post-operative hospital stay, first rank indicates highest probability of hospitalization. 
Although reconstruction techniques after distal gastrectomy was a very hot spot issue in recent years, which one is the best has not been proved by any trial or research yet. We tried to make a conclusion of all these related researches. Moreover, we also organized a perspective clinical trial to compare the URY and BII anastomosis (Clinicaltrials.gov: NCT02763878), and the early short-term result showed that URY have a great advantage in reducing anti-reflux symptoms, and improving patient's quality of life after surgery. Therefore, we believed that more attentions should be paid on URY which may improve patients' QOL. Unfortunately, after enrolling all the studies, we only found one study referred to URY which meet our inclusion criteria. Then we had to analyze data with and without URY separately, finally we found out that there was no significant difference whether including URY or not. However, URY still showed a better tendency to improve post-operative reflux phenomenon than BII despite of only one research was involved. We believed that this would be an important prompt to later researches. And we believed, with the amounts of URY studies increasing, the superiority of URY in reducing anti-reflux and post-operative complications would be eventually shown. Based on our findings, we suggested that later studies should focus on the following points. First, more researches or trials should be focused on the comparison of URY and other reconstruction techniques. Second, regarding its safety, we need pay more attentions on the postoperative anastomotic leakage, anastomotic bleeding and so on. And at last, economic benefits are also need to be taken into consideration.

There were still limitations in our study. Above all, our net-work meta-analysis included some retrospective studies, which may make the degree of evidence equal to a retrospective clinical trial at best. Additionally, the methods to diagnose the parameters we were concerned with varied, which may have led to a data extraction bias. Nonetheless, our study provides information for surgeons when they decide on an optimal reconstruction technique for their patients.

\section{Conclusion}

We realize that the ideal treatment option may differ according to the parameters prioritized by surgeons and patients, and the truth is that the optimal method should not only minimize postoperative morbidity but also improve quality of life. Despite the limitations, our indirect comparison suggested that RY is superior to other reconstruction skills in preventing reflux symptoms and that URY, with high expectations, should take the place of RY in the future. Further RCTs are required to confirm the safety and efficacy of the URY reconstruction technique compared to RY.

\section{Acknowledgments}

This work is supported by the Youth Cultivation Project of Sun Yat-sen University (17ykpy65) and 5010 Project of Sun Yat-sen University (2012017).

\section{Competing Interests}

The authors have declared that no competing interest exists.

\section{References}

1. Hu J, Zhao Y, Ren M, Li Y, Lu X, Lu G, et al. The Comparison between Endoscopic Submucosal Dissection and Surgery in Gastric Cancer: A Systematic Review and Meta-Analysis. Gastroenterol Res Pract. 2018;2018:4378945

2. Coburn N, Cosby R, Klein L, Knight G, Malthaner R, Mamazza J, et al. Staging and surgical approaches in gastric cancer: a clinical practice guideline. Current oncology (Toronto, Ont). 2017;24(5):324-31.

3. Zong L, Chen P. Billroth I vs. Billroth II vs. Roux-en-Y following Distal Gastrectomy: A Meta-Analysis Based on 15 Studies. Hepato-Gastroenterology. 2011;58(109):1413-24.

4. Xiong JJ, Altaf K, Javed MA, Nunes QM, Huang W, Mai G, et al. Roux-en-Y versus Billroth I reconstruction after distal gastrectomy for gastric cancer: a meta-analysis. World journal of gastroenterology. 2013;19(7):1124-34.

5. Montesani C, D'Amato A, Santella S, Pronio A, Giovannini C, Cristaldi M, et al. Billroth I versus Billroth II versus Roux-en-Y after subtotal gastrectomy. Perspective randomized study. Hepato-Gastroenterology. 2002;49(47):1469-73.

6. Nakada K, Takahashi M, Ikeda M, Kinami S, Yoshida M, Uenosono Y, et al. Factors affecting the quality of life of patients after gastrectomy as assessed using the newly developed PGSAS-45 scale: A nationwide multi-institutional study. World journal of gastroenterology. 2016;22(40):8978-90.

7. Ishikawa M, Kitayama J, Kaizaki S, Nakayama H, Ishigami H, Fujii S, et al. Prospective randomized trial comparing Billroth I and Roux-en-Y procedures after distal gastrectomy for gastric carcinoma. World journal of surgery. 2005; 29(11):[1415-20; discussion 21 pp.].

8. Gavini S, Vutukuru V, Radhakrishna K, Parthasarathy S. Prospective randomised study comparing billroth ii with Braun anastomosis versus roux-en-y reconstruction after radical distal gastrectomy. United European Gastroenterology Journal. 2016;4(5):A236-A7.

9. Zhang YM, Liu XL, Xue DB, Wei YW, Yun XG. Myoelectric activity and motility of the Roux limb after cut or uncut Roux-en-Y gastrojejunostomy. World journal of gastroenterology. 2006;12(47):7699-704.

10. Frankel LA. Roux stasis syndrome: treatment by pacing and prevention by use of an 'uncut' Roux limb. Archives of surgery (Chicago, Ill : 1960). 1992;127(9):1135-6.

11. Tu BN, Kelly KA. Elimination of the Roux stasis syndrome using a new type of "uncut Roux" limb. American journal of surgery. 1995;170(4):381-6.

12. Uyama I, Sakurai Y, Komori Y, Nakamura Y, Syoji M, Tonomura S, et al. Laparoscopy-assisted uncut Roux-en-Y operation after distal gastrectomy for gastric cancer. Gastric cancer : official journal of the International Gastric Cancer Association and the Japanese Gastric Cancer Association. 2005;8(4):253-7.

13. Shibata C, Kakyo M, Kinouchi M, Tanaka N, Miura K, Naitoh T, et al. Results of modified uncut Roux-en-Y reconstruction after distal gastrectomy for gastric cancer. Hepato-gastroenterology. 2013;60(127):1797-9.

14. Van Stiegmann G, Goff JS. An alternative to Roux-en-Y for treatment of bile reflux gastritis. Surgery, gynecology \& obstetrics. 1988;166(1):69-70.

15. Hozo SP, Djulbegovic B, Hozo I. Estimating the mean and variance from the median, range, and the size of a sample. BMC Medical Research Methodology. 2005;5(1).

16. Chareton B, Landen S, Manganas D, Meunier B, Launois B. Prospective randomized trial comparing Billroth I and Billroth II procedures for carcinoma of the gastric antrum. Journal of the American College of Surgeons. 1996;183(3):190-4.

17. Imamura H, Takiguchi S, Yamamoto K, Hirao M, Fujita J, Miyashiro I, et al. Morbidity and mortality results from a prospective randomized controlled trial comparing Billroth I and Roux-en-Y reconstructive procedures after distal gastrectomy for gastric cancer. World journal of surgery. 2012;36(3):632-7.

18. Lee MS, Ahn SH, Lee JH, Park DJ, Lee HJ, Kim HH, et al. What is the best reconstruction method after distal gastrectomy for gastric cancer? Surgical endoscopy. 2012;26(6):1539-47.

19. Takiguchi S, Yamamoto K, Hirao M, Imamura H, Fujita J, Yano M, et al. A comparison of postoperative quality of life and dysfunction after Billroth I and Roux-en-Y reconstruction following distal gastrectomy for gastric cancer: 
results from a multi-institutional RCT. Gastric cancer : official journal of the International Gastric Cancer Association and the Japanese Gastric Cancer Association. 2012;15(2):198-205

20. Nakamura M, Nakamori M, Ojima T, Iwahashi M, Horiuchi T, Kobayashi Y, et al. Randomized clinical trial comparing long-term quality of life for Billroth I versus Roux-en-Y reconstruction after distal gastrectomy for gastric cancer. British journal of surgery. 2016; 103(4):[337-47 pp.].

21. Choi YY, Noh SH, An JY. A randomized controlled trial of Roux-en-Y gastrojejunostomy vs. gastroduodenostomy with respect to the improvement of type 2 diabetes mellitus after distal gastrectomy in gastric cancer patients. PloS one. 2017; 12(12):[e0188904 p.].

22. So JB, Rao J, Wong AS, Chan YH, Pang NQ, Tay AY, et al. Roux-en-Y or Billroth II Reconstruction After Radical Distal Gastrectomy for Gastric Cancer: A Multicenter Randomized Controlled Trial. Annals of surgery. 2017.

23. Yang D, He L, Tong WH, Jia ZF, Su TR, Wang Q. Randomized controlled trial of uncut Roux-en-Y vs Billroth II reconstruction after distal gastrectomy for gastric cancer: Which technique is better for avoiding biliary reflux and gastritis? World journal of gastroenterology. 2017;23(34):6350-6.

24. Yang K, Zhang WH, Liu K, Chen XZ, Zhou ZG, Hu JK. Comparison of quality of life between Billroth-I and Roux-en-Y anastomosis after distal gastrectomy for gastric cancer: A randomized controlled trial. Scientific reports. 2017;7.

25. Nunobe S, Okaro A, Sasako M, Saka M, Fukagawa T, Katai H, et al. Billroth 1 versus Roux-en-Y reconstructions: a quality-of-life survey at 5 years. International journal of clinical oncology. 2007;12(6):433-9.

26. Kojima K, Yamada H, Inokuchi M, Kawano T, Sugihara K. A comparison of Roux-en-Y and Billroth-I reconstruction after laparoscopy-assisted distal gastrectomy. Annals of surgery. 2008;247(6):962-7.

27. Namikawa $T$, Kitagawa $H$, Okabayashi $T$, Sugimoto $T$, Kobayashi M, Hanazaki K. Roux-en-Y reconstruction is superior to billroth I reconstruction in reducing reflux esophagitis after distal gastrectomy: special relationship with the angle of his. World journal of surgery. 2010;34(5):1022-7.

28. Kang KC, Cho GS, Han SU, Kim W, Kim HH, Kim MC, et al. Comparison of Billroth I and Billroth II reconstructions after laparoscopy-assisted distal gastrectomy: a retrospective analysis of large-scale multicenter results from Korea. Surgical endoscopy. 2011;25(6):1953-61.

29. Kumagai K, Hiki N, Nunobe S, Jiang X, Kubota T, Aikou S, et al. Different features of complications with Billroth-I and Roux-en-Y reconstruction after laparoscopy-assisted distal gastrectomy. Journal of gastrointestinal surgery : official journal of the Society for Surgery of the Alimentary Tract. 2011;15(12):2145-52.

30. Tanaka S, Matsuo K, Matsumoto H, Maki T, Nakano M, Sasaki T, et al. Clinical outcomes of Roux-en-Y and Billroth I reconstruction after a distal gastrectomy for gastric cancer: What is the optimal reconstructive procedure? Hepato-gastroenterology. 2011;58(105):257-62.

31. Lee SW, Tanigawa N, Nomura E, Tokuhara T, Kawai M, Yokoyama K, et al. Benefits of intracorporeal gastrointestinal anastomosis following laparoscopic distal gastrectomy. World journal of surgical oncology. 2012;10:267.

32. Inokuchi M, Kojima K, Yamada H, Kato K, Hayashi M, Motoyama K, et al. Long-term outcomes of Roux-en-Y and Billroth-I reconstruction after laparoscopic distal gastrectomy. Gastric cancer : official journal of the International Gastric Cancer Association and the Japanese Gastric Cancer Association. 2013;16(1):67-73.

33. An JY, Cho I, Choi YY, Kim YM, Noh SH. Totally laparoscopic Roux-en-Y gastrojejunostomy after laparoscopic distal gastrectomy: analysis of initial 50 consecutive cases of single surgeon in comparison with totally laparoscopic Billroth I reconstruction. Yonsei medical journal. 2014;55(1):162-9.

34. Shim JH, Oh SI, Yoo HM, Jeon HM, Park CH, Song KY. Roux-en-Y gastrojejunostomy after totally laparoscopic distal gastrectomy: comparison with Billorth II reconstruction. Surgical laparoscopy, endoscopy \& percutaneous techniques. 2014;24(5):448-51.

35. Komatsu S, Ichikawa D, Kubota T, Okamoto K, Shiozaki A, Fujiwara H, et al. Clinical outcomes and quality of life according to types of reconstruction following laparoscopy-assisted distal gastrectomy for gastric cancer. Surgical laparoscopy, endoscopy \& percutaneous techniques. 2015;25(1):69-73.

36. Imamura T, Komatsu S, Ichikawa D, Kosuga T, Okamoto K, Konishi H, et al. Reconstruction method as an independent risk factor for the postoperative decrease in hemoglobin in stage I gastric cancer. Journal of gastroenterology and hepatology. 2016;31(5):959-64.

37. Tran TB, Worhunsky DJ, Squires MH, Jin LX, Spolverato G, Votanopoulos KI, et al. To Roux or not to Roux: a comparison between Roux-en-Y and Billroth II reconstruction following partial gastrectomy for gastric cancer. Gastric cancer : official journal of the International Gastric Cancer Association and the Japanese Gastric Cancer Association. 2016;19(3):994-1001.

38. Virgilio E, Balducci G, Mercantini P, Ferri M, Bocchetti T, Caterino S, et al. Reconstruction After Distal Gastrectomy for Gastric Cancer: Billroth 2 or Roux-En-Y Procedure? Anticancer research. 2017;37(10):5595-602.

39. Park JY, Kim YJ. Uncut Roux-en-Y Reconstruction after Laparoscopic Distal Gastrectomy Can Be a Favorable Method in Terms of Gastritis, Bile Reflux, and Gastric Residue. Journal of gastric cancer. 2014;14(4):229-37. 